\title{
Hepatic Rupture Complicating HELLP Syndrome in Pregnancy
}

\author{
Lt Col Y Singh*, Col SPS Kochar ${ }^{+}$, Col M Biswas*, Lt Col KJ Singh ${ }^{* *}$
}

MJAFI 2009; 65 : 89-90

Key Words: Hepatic rupture; Pregnancy; HELLP syndrome; Preeclampsia

\section{Introduction}

Subcapsular hematoma and hepatic rupture are very Uunusual catastrophic complication of preeclampsia/ eclampsia and HELLP (hemolysis, elevated liver enzymes, and low platelets) syndrome [1]. The reported incidence of this condition varies from 1 in 40,000 to 1 in 2,50,000 deliveries [2]. There is no agreement on the best approach to treat this severe complication of pregnancy and optimal management is still evolving. A multidisciplinary approach to the management of these patients can lead to remarkable decrease in the usual high mortality rate. We present a fatal case of severe preeclampsia, which rapidly progressed to HELLP syndrome, liver rupture, disseminated intravascular coagulation (DIC) and renal failure.

\section{Case Report}

A 22 years old second gravida lady at 34 weeks of gestation was admitted for preeclampsia. All her investigations (except for proteinuria of 1+) including antenatal sonography, non stress test (NST) and fundoscopy were normal. She had normal delivery two years back with no antenatal/post natal complications. On the next day of admission she had rapid progression to severe preeclampsia and it was decided to terminate pregnancy with cervical application of prostaglandin E2 (PGE2) gel. She was put on anti hypertensives and magnesium sulfate (Pritchard regime). Magnesium sulphate was given by standard Pritchard intermittent intramuscular schedule : 4 gm as slow intravenous $(20 \%)$ at the rate of $1 \mathrm{gm} /$ minute along with $10 \mathrm{gm}$ of $50 \%$ magnesium sulfate intramuscular, $5 \mathrm{gm}$ of $50 \%$ every four hour (total two doses) were given. Injection labetalol hydrochloride $20 \mathrm{mg}$ by slow intravenous route over two minutes was given initially and was repeated twice in the doses of $40 \mathrm{mg}$ and $80 \mathrm{mg}$ at intervals of 10 minute as the desired effect was not achieved. Blood pressure ranged between 150/94 $\mathrm{mm} \mathrm{Hg}$ and 180/130 $\mathrm{mm} \mathrm{Hg}$. When the patient was in early labor she developed hematuria, oliguria and features of imminent eclampsia, including epigastric pain. Urgent investigations revealed platelet count of 90,000 c/ $\mathrm{mm}$, proteinuria of $3+$ and elevated liver transaminases (aspartate aminotransferase was $82 \mathrm{IU} / \mathrm{L} \&$ alanine aminotransferase $84 \mathrm{IU} / \mathrm{L})$. Serum bilirubin was $1.8 \mathrm{mg} / \mathrm{dL}$ and peripheral blood showed evidence of hemolysis. Since there was no satisfactory progress of labor it was decided to terminate pregnancy by cesarean section. While patient was awaiting surgery she had eclamptic seizures (having received last dose of magnesium sulphate about two hours back). The seizures were followed by sudden fall in blood pressure, cutaneous pallor and altered mental status. After resuscitation cesarean section was done and a fresh stillborn baby weighing $2.2 \mathrm{~kg}$ delivered. The patient had cardiopulmonary arrest during surgery. There was massive hemoperitoneum and about 3 liters of blood/ clots were removed. There was active bleeding from visceral (inferior) surface of liver with marked congestion. A subcapsular rupture of the liver was identified. Tight packing with roller gauze was done which controlled bleeding. During peroperative period she developed DIC (serum fibrinogen was $86 \mathrm{mg} / \mathrm{dL}$, D-Dimers were positive) and had atonic post partum hemorrhage which was managed by using prostaglandins and internal iliac artery ligation. B-Lynch brace sutures for uterine compression were also done to minimize uterine bleeding. In the immediate postoperative period, patient developed acute respiratory distress syndrome and renal failure. In spite of blood component and supportive therapy the patient died 18 hours after surgery.

\section{Discussion}

Inspite of improvement in antenatal care maternal mortality in developing countries is high. Hypertensive disorders, including HELLP syndrome are one of the main causes of maternal mortality [3]. HELLP syndrome is a disease of variable presentation with high mortality and morbidity [4]. Liver rupture and hemorrhage is the most unusual and serious complication of HELLP syndrome [5]. The cause of subcapsular and

*Associate Professor, +Professor \& Head, "Professor (Department of Obstetrics \& Gynaecology), **Associate Professor (Department of Surgery), AFMC, Pune.

Received : 03.03.08; Accepted :11.10.08_E-mail: yoginaral@yahoo.co.uk 
intraparenchymal hepatic hematoma in HELLP syndrome is not definitely known. A florescent antibody technique has been used to demonstrate fibrin deposition on basement membrane of hepatic sinusoids and arterioles of eclamptic patients. It has been postulated that sensitization of the reticuloendothelial system of the liver by preeclampsia may render it unable to clear fibrin thrombi from the circulation. As a result, infarction with vascular disruption may develop leading to intrahepatic hemorrhage and parenchymal destruction. Rarely the periportal necrosis that result coalesces and forms sub capsular hematoma, leading to rupture of Glissons capsule with intraperitoneal hemorrhage as was seen in our patient. Specific symptoms can be established through identification of a triad; epigastric/right upper quadrant pain, hypotension without evident cause bleeding and clinical data of hypertensive disease of pregnancy.

Ultrasound scan is the quickest means of diagnosis although computerized tomography is more sensitive. Magnetic resonance imaging (MRI) is an alternate option used in a less urgent situation or in diagnosing a pregnant patient. The management of contained hematoma is to support the patient, with surgery reserved for those who are hemodynamically unstable or documented expansion of hematoma. Various surgical treatments have been described, depending on the severity of rupture. Surgical treatment can encompass packing of the bleeding surface with absorbable gelatin sponge and resection. When the bleeding cannot be contained and acute liver failure occurs, liver transplantation has been reported. Argon beam coagulator for treatment of hepatic rupture due to HELLP syndrome has been reported [7]. Recombinant factor VIIa has been used with success to help control bleeding in HELLP syndrome [8]. Finally a multidisciplinary approach to the management of these patients can lead to remarkable decrease in the high mortality rate.

Conflicts of Interest

None identified

References

1. Sheikh RA, Yasmeen S, Pauly MP, RieglerJL. Spontaneous intrahepatic hemorrhage and hepatic rupture in HELLP syndrome: four cases and a review. J Clin Gastroenterol 1999;28:323-8.

2. Corinna W, Pereira P, Neeser E, et al. Subcapsular liver hematoma in HELLP syndrome: Evaluation of diagnostic and intrahepatic options-A unicentric study. Am J Obstet Gynecol 2004;190:106-12.

3. Ana CPF Araujo, Marcos D Leao, Maria H Nobrega, et al. Characteristics and treatment of hepatic rupture caused by HELLP syndrome. Am J Obstet Gynecol 2006;195:129-33.

4. Aldemir M, Bac B, Tacyldiz, Yagmur Y, Kelesc. Spontaneous liver hematoma and hepatic rupture in HELLP syndrome: report of two cases. Surg Today 2002;32:450-3.

5. Catriconi M, Aragiusto G, Ansalonem. Liver rupture in HELLP syndrome. Report of a case. Minerva Chir 2000;55:167-71.

6. Boormans EM, Bekedan DJ, Lenters E, et al. Spontaneous liver hemorrhage during pregnancy: a rare and life threatening situation. Ned Tijdschr Geneeskd 2007;151:1157-62.

7. Shrivastava VK, Imagaead D, Wing A. Argon beam coagulator for treatment of hepatic rupture with HELLP syndrome. Obstet Gynecol 2006; 107: 525-6.

8. Dart BW $4^{\text {th }}$, Cockerham WT, Torres C, et al. A novel use of recombinant factor VIIa in HELLP syndrome associated with spontaneous hepatic rupture and abdominal compartment syndrome. J Trauma 2004; 57:171-4. 